\title{
The formation of massive black holes through collision runaway in dense young star clusters
}

\author{
Simon F. Portegies Zwart \\ Astronomical Institute 'Anton Pannekoek', \\ University of Amsterdam, Kruislaan 403 \\ and \\ Institute for Computer Science, \\ University of Amsterdam, Kruislaan 403 \\ spz@science.uva.nl \\ Holger Baumgardt \\ Institute of Advanced Physical and Chemical Research RIKEN, \\ 2-1 Hirosawa, Wako-shi, Saitama 351-019, Japan \\ Piet Hut \\ Institute for Advanced Study, Princeton, NJ 08540, USA \\ Junichiro Makino \\ Department of Astronomy, University of Tokyo, Tokyo 113, Japan \\ Stephen L. W. McMillan \\ Department of Physics, Drexel University, \\ Philadelphia, PA 19104, USA
}

May 29, 2018

A luminous X-ray source is associated with a cluster (MGG-11) of young stars $\sim 200$ pc from the center of the starburst galaxy M82 ${ }^{1,2}$. The properties of the X-ray source are best explained by a black hole with a mass of at least $350 \mathrm{M}_{\odot}{ }^{3,4}$, which is intermediate between stellar-mass and supermassive black holes. A 
nearby but somewhat more massive star cluster (MGG-9) shows no evidence of such an intermediate mass black hole ${ }^{1,3}$, raising the issue of just what physical characteristics of the clusters can account for this difference. Here we report numerical simulations of the evolution and the motions of stars within the clusters, where stars are allowed to mergers with each other. We find that for MGG-11 dynamical friction leads to the massive stars sinking rapidly to the center of the cluster to participate in a runaway collision, thereby producing a star of $800-3000 \mathrm{M}_{\odot}$, which ultimately collapses to an black hole of intermediate mass. No such runaway occurs in the cluster MGG-9 because the larger cluster radius leads to a mass-segregation timescale a factor of five longer than for MGG-11.

Using the Keck NIRSPEC spectrometer, McCrady et al. ${ }^{1}$ have made accurate measurements of the bulk parameters of MGG-11 and MGG-9, two of the brightest star clusters in the central region of M82. Figure 1 shows $\mathrm{X}$-ray ${ }^{3}$ and near-infrared ${ }^{1}$ images $^{2}$ of the area of interest around the two clusters. The relative positional accuracies of both sets of observations are better than 1 arcsecond. However, the absolute pointing accuracy is much poorer, for both telescopes. Although apparently off-center, the positions of the star cluster MGG-11 and the bright X-ray source are in fact consistent with one another (D. Pooley, private communication).

Both MGG-9 and MGG-11 have quite similar ages, in the range $7-12 \mathrm{Myr}{ }^{1}$. The line-of-sight velocity dispersion of MGG-11 $\left(\sigma_{r}=11.4 \pm 0.8 \mathrm{~km} \mathrm{~s}^{-1}\right)$ is somewhat smaller than that of MGG-9 $\left(\sigma_{r}=15.9 \pm 0.8 \mathrm{~km} \mathrm{~s}^{-1}\right)$. Combining these numbers with the projected half-light radii, $r=1.2 \mathrm{pc}$ for MGG-11 and $r=2.6 \mathrm{pc}$ for MGG-9, McCrady et al. estimate a total cluster mass of $\sim(3.5 \pm 0.7) \times 10^{5} \mathrm{M}_{\odot}$ for $\mathrm{MGG}-11$, and about four times higher for MGG-9.

In young dense clusters like MGG-11, supermassive stars may form through repeated collisions $6,7,8$. The collision rate will be greatly enhanced if massive stars have time to reach the core before exploding as supernovae ${ }^{9}$. Dynamical friction implies a characteristic time scale $t_{\mathrm{df}}$ for a massive star in a roughly circular orbit to sink from the half-mass radius $R$ to the cluster center ${ }^{10}$ :

$$
t_{\mathrm{df}} \simeq \frac{\langle m\rangle}{100 \mathrm{M}_{\odot}} \frac{0.138 N}{\ln \left(0.11 M / 100 \mathrm{M}_{\odot}\right)}\left(\frac{R^{3}}{G M}\right)^{1 / 2} .
$$

Here $\langle m\rangle$ and $M$ are the mean stellar mass and the total mass of the cluster, respectively, $N$ is the number of stars, and $G$ is the gravitational constant. For definiteness, we have evaluated $t_{\mathrm{df}}$ for a $100 \mathrm{M}_{\odot}$ star. Less massive stars undergo weaker dynamical friction, and thus must start at smaller radii in order to reach the cluster center on a similar time scale.

For MGG-11, we find $t_{\mathrm{df}} \sim 3 \mathrm{Myr}$, which is comparable to the main-sequence lifetimes of the most massive stars. On the other hand, for MGG-9, $t_{\mathrm{df}} \sim 15$ Myr. Thus, massive stars in MGG-11 can easily reach the center of the cluster before exploding as supernovae, whereas those in MGG-9 do not. Given the high central 
density of MGG-11, its massive stars, once accumulated in the cluster center, cannot avoid a runaway collision ${ }^{6}$.

We have tested this scenario by carrying out star-by-star simulations of MGG11 and MGG-9 using two independently developed $N$-body codes, Starlab ${ }^{15}, 16$ and NBODY4 17, 18. A more extensive discussion of those simulations is presented as Supplementary Information. Briefly, we choose the initial conditions of our model clusters so that today, at an age of 7-12 Myr, they have mass functions, luminosities, half-mass radii and velocity dispersions in agreement with the McCrady et al. observations. Since we do not know either the initial or the current central densities of either cluster, the concentration $c$ is treated as a free parameter controlling the initial central density of our model clusters. [The concentration parameter is defined as the ratio of the core radius to the tidal radius: $c \equiv \log \left(R_{\mathrm{t}} / R_{\mathrm{c}}\right)$.]

We find that, for $c>2$ (which for "King" 15 models is equivalent to a dimensionless central potential $W_{0} \gtrsim 9$ ) our MGG-11 models do indeed show runaway growth via repeated collisions. Figure 2 presents, for several such simulations, the growth in mass of the star that would ultimately become the most massive star in the cluster. We will refer to this star simply as "the runaway star". Based on detailed supernova calculations, we assume that stars having masses $>260 \mathrm{M}_{\odot}$ collapse to black holes without significant mass loss in supernova explosions ${ }^{16}$. Our stellar evolution models for stars with masses between 50 and $1000 \mathrm{M}_{\odot}$ are based on detailed calculations for such high-mass stars ${ }^{34,33}$.

The quantitative differences evident in Figure 2 between the simulations performed with Starlab and those using NBODY4 are due mainly to the different radii assumed for the runaway star in those two packages. Stars of masses $\gtrsim 100 \mathrm{M}_{\odot}$ in NBODY4 are larger (in a time-averaged sense) by about a factor of 3 compared to those in Starlab. (More details are provided in Supplementary Information.) Because gravitational focusing dominates the collision cross section, this difference propagates linearly in the collision rate, explaining the factor $\sim 3$ difference in the final mass of the runaway star. Apart from this effect, we find that our qualitative results are quite insensitive to the details of the adopted evolution prescription.

For MGG-11, the runaway star typically experienced a total of $\sim 10-100$ collisions. Most of them occurred during the first $3 \mathrm{Myr}$, that is, before the star became a black hole. The collision counterparts are usually $30-50 \mathrm{M}_{\odot}$ main-sequence stars. By the time the runaway star collapsed to a black hole it had reached a mass of $800-3000 \mathrm{M}_{\odot}$. Later the black hole may capture a companion star to become an ultraluminous X-ray source ${ }^{19}$.

No episode of runaway growth was seen in our MGG-11 models with $c<2$, nor in any of the MGG-9 simulations, regardless of initial concentration. Thus we see very clearly that differences in bulk parameters, specifically the dynamical friction time scale for the most massive stars, can readily explain why MGG-11 might host an IMBH while MGG-9 does not. Simulations of MGG-11 with $c>2\left(W_{0} \gtrsim 9\right)$ reached core collapse 20 before 1 Myr. Models with $c<2\left(W_{0} \lesssim 8\right)$ also showed some increase in central density, but the maximum density was much lower (and occurred later) because the collapse was stopped by supernova mass loss after $\sim 3$ Myr. Thus, a collision runaway could not occur in these clusters. 
Figure 3 summarizes the results of our simulations, illustrating how both high initial concentration and short dynamical friction time scales are needed to lead to a collision runaway. In addition to the parameters for MGG-9 and MGG-11, we also indicate on the figure the location of the young star cluster [W99]121, one of several star clusters in the Antennae system for which accurate structure parameters have been determined ${ }^{9}$. Its parameters are quite similar to those of MGG-9. The cluster [W99]1 has a bright $L_{0.2-10 \mathrm{keV}} \simeq 10^{38.7} \mathrm{erg} / \mathrm{s}$ X-ray point source as a counterpart, ${ }^{23}$ the luminosity of which is consistent with a "normal" high-mass X-ray binary containing a magnetized neutron star or a stellar-mass black hole ${ }^{23}$. Star clusters such as MGG-11, MGG-9 and [W99]1 are richly populated with black holes and neutron stars - based on our adopted mass function, we expect these clusters to contain some 1400 stellarmass black holes and up to about 1000 neutron stars. The formation of an ordinary high-mass X-ray binary is therefore not surprising.

The relevant parameters of five other young clusters in the Antennae ${ }^{9}$ all fall far to the right of Figure 3 and are therefore not expected to contain IMBHs. This is consistent with the absence of ultraluminous X-ray sources in these clusters. The Milky Way galaxy contains (at least) four young dense star clusters, of which the Arches, Quintuplet, and NGC 3603 have the right conditions for multiple stellar collisions to occur. However, the relatively small number of stars in these systems may prevent the growth of an object massive enough to collapse to an IMBH. In any case, these clusters are currently too young to have experienced any supernovae. The slightly older star cluster Westerlund $1^{24}$ is sufficiently massive, and fulfills the criteria for producing an IMBH via the process described above. So far, no bright $\mathrm{X}$-ray source has been found in this cluster.

Based on theoretical considerations, supported by extensive numerical simulations, we have shown that an IMBH of mass $\sim 800-3000 \mathrm{M}_{\odot}$ is expected to form through runaway collisions in the star cluster MGG-11, but not in MGG-9. The requirement for the formation of such an IMBH in MGG-11 is that the cluster was born with $c \gtrsim 2$ and $t_{\mathrm{df}} \lesssim 4 \mathrm{Myr}$. While high by the standards of typical open and globular clusters observed in our Galaxy, such a density is not uncommon among young ( $\lesssim 3$ Myr old) star clusters. Examples include NGC $3603(c \geq 2.08)^{25}$ in the Milky Way Galaxy, and $\mathrm{R} 136$ in the 30 Doradus region of the LMC ${ }^{26}$. We propose that the IMBH thus formed is the origin of the $L_{0.2-10 \mathrm{keV}} \simeq 10^{41} \mathrm{erg} / \mathrm{s}$ ultraluminous X-ray point source seen at the position of MGG-11. 
1. Matsumoto, H., Tsuru, T. G. X-ray evidence of an AGN in M82. Pub. Astron. Soc. Jap. 51, 321-331 (1999) [erratum in Pub. Astron. Soc. Jap. 51, 567 (1999)]

2. Kaaret. $\mathrm{P}$, et al. Chandra high-resolution camera observations of the luminous $\mathrm{X}$-ray source in the starburst galaxy M82. Mon. Not. R. Astron. Soc. 321, L29-L32 (2001).

3. Matsumoto, $\mathrm{H}$. et al. Discovery of a luminous, variable, off-center source in the nucleus of M82 with the Chandra high-resolution camera. Astrophys. J. 547, L25-L28 (2001).

4. Strohmayer, T. E., Mushotsky, R. F. Discovery of X-ray quasi-periodic oscillations from an ultraluminous X-ray source in M82: Evidence against beaming. Astrophys. J. 586, L61-L64 (2003).

5. McCrady, N., Gilbert, A. M., Graham, J. R. Kinematic masses of super star clusters in M82 from high-resolution near-infrared spectroscopy. Astrophys. J. 596, 240-252 (2003).

6. Portegies Zwart, S. F., Makino, J., McMillan, S. L. W., Hut, P. Star cluster ecology. III. Runaway collisions in young compact star clusters. Astron. Astrophys. 348, 117-126 (1999).

7. Portegies Zwart, S. F. \& McMillan, S. L. W. The runaway growth of intermediate-mass black holes in dense star clusters. Astrophys. J. 576, 899-907 (2002).

8. Gürkan, M. A., Freitag, M., Rasio, F. A. Formation of massive black holes in dense star clusters. I. Mass segregation and core collapse. Accepted for publication in Astrophys. J. http://arxiv.org/list/astro-ph/0308449 (2003).

9. Ebisuzaki, T. et al. Missing link found? The "runaway" path to supermassive black holes. Astrophys. J. 562, L19-L22 (2001).

10. Spitzer, L. Jr., Hart, M. H. Random gravitational encounters and the evolution of spherical systems. I. Method. Astrophys. J. 164, 399-409 (1971).

11. Portegies Zwart, S. F., McMillan, S. L. W., Hut, P., Makino, J. Star cluster ecology - IV. Dissection of an open star cluster: photometry. Mon. Not. R. Astron. Soc. 321, 199-226 (2001).

12. Hut, P., Makino, J., McMillan, S. L. W., Portegies Zwart S. Starlab. http://manybody.org/manybody/starlab.html (1995)

13. Aarseth, S. J. From NBODY1 to NBODY6: The growth of an industry. Pub. Astron. Soc. Pac. 111, 1333-1346 (1999).

14. Baumgardt, H., Makino, J. Dynamical evolution of star clusters in tidal fields. Mon. Not. R. Astron. Soc. 340, 227-246 (2003). 
15. King, I. R. The structure of star clusters. III. Some simple dynamical models. Astron. J. 71, 64-75 (1966).

16. Heger, A., Fryer, C. L., Woosley, S. E., Langer, N., Hartmann, D. H. How massive single stars end their life. Astrophys. J. 591, 288-300 (2003).

17. Stothers, B., Chin, C-W. On two theories of the cyclical outbursts of eta Carinae. Astrophys. J. 489, 319 (1997).

18. Ishii, M., Munetaka, U., Kato, M. Core-halo structure of a chemically homogeneous massive star and bending of the zero-age main sequence. Pub. Astron. Soc. Jap. 51, 417 (1999).

19. Hopman, C., Portegies Zwart, S. F., Alexander, T. Ultraluminous X-ray sources as intermediate mass black holes fed by tidally captured stars. Submitted to Astrophys. J. Letters, http://arxiv.org/list/astro-ph/0312597 (2003).

20. Heggie, D. C., Hut, P. The Gravitational Million-Body Problem Cambridge University Press, Cambridge (2003).

21. Whitmore, B. C. et al. The luminosity function of young star clusters in "The Antennae" galaxies. Astrophys. J. 118, 1551-1576 (1999).

22. Mengel, S., Lehnert, M. D., Thatte, N., Genzel, R. Dynamical masses of young star clusters in NGC 4038/4039. Astron. Astrophys. 383, 137-152 (2002).

23. Zezas, A., Fabbiano, G., Rots, A. H., Murray, S. S. Chandra observations of "The Antennae" galaxies (NGC 4038/4039). III. X-ray properties and multiwavelength associations of the X-ray source population. Astrophys. J. 577, 710-725 (2002).

24. Piatti, A. E., Bica, E., Clariá, J.J. Fundamental parameters for the highly reddened young open clusters Westerlund 1 and 2. Astron. Astrophys. Supplement 127, 423-432 (1998).

25. Moffat, A. F. J., Drissen, L., Shara, M., M. NGC 3603 and its Wolf-Rayet stars: Galactic clone of R136 at the core of 30 Doradus, but without the massive surrounding cluster halo. Astrophys. J. 436, 183-193 (1994).

26. Campbell, B. et al. Hubble Space Telescope planetary camera images of R136. Astron. J. 104, 1721-1742 (1992).

27. Matsushita, M. S. et al. Formation of a massive black hole at the center of the superbubble in M82. Astrophys. J. 545, L107-L111 (2000).

28. Salpeter, E., E. The luminosity function and stellar evolution. Astrophys. J. 121, 161-167 (1955).

29. Kroupa, P. On the variation of the initial mass function. Mon. Not. R. Astron. Soc. 322, 231-246 (2001) 
We are grateful to Nate McCrady and Dave Pooley for discussions on MGG-11, to Takeshi Go Tsuru for an accurate position of M82 X-1 and to Edward van den Heuvel for critically reading the manusctipt. This work was supported by NASA ATP, the Royal Netherlands Academy of Sciences (KNAW), the Dutch organization of Science (NWO), and by the Netherlands Research School for Astronomy (NOVA).

Correspondence and requests for materials should be addressed to Simon Portegies Zwart (email: spz@science.uva.nl) 


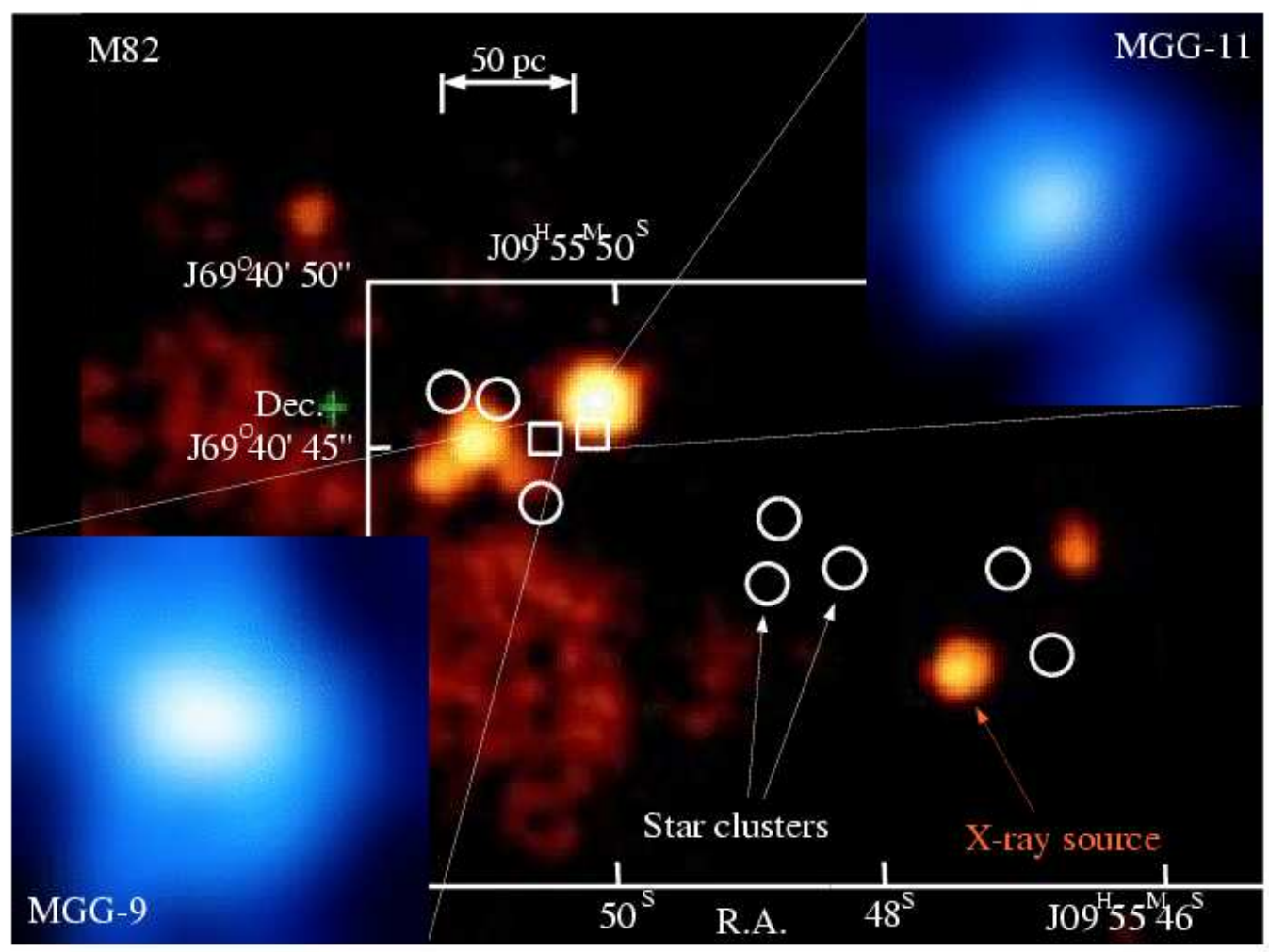

Figure 1: Chandra X-ray image (in R.A. and Dec.) of the relevant part of the starburst galaxy M82 with the observed star clusters indicated. The color image is from the 28 October 1999 X-ray observation by Matsumoto et al. (2001), ${ }^{3}$ with about arcsecond positional accuracy. The brightest X-ray source (M82 X-1) is near the center of the image. The star clusters, from table 3 of McCrady et al. (2003), ${ }^{1}$ are indicated by circles. The positions of the two star clusters MGG-9 and MGG-11 are indicated with squares. The magnified infrared images of these star clusters from the McCrady et al observations are presented in the upper right (MGG-11) and lower left (MGG-9) corners. A recently discovered $54.4 \pm 0.9 \mathrm{mHz}$ quasi-periodic oscillator is not shown because of its low ( 7 arcsecond) positional accuracy ${ }^{4}$, but its position is consistent with the X-ray source in MGG-11. A millimeter source 27 roughly centered around the two clusters is not shown either, because it is a large shell-like structure with a diameter of $\sim 14^{\prime \prime} \times 9^{\prime \prime}$. 


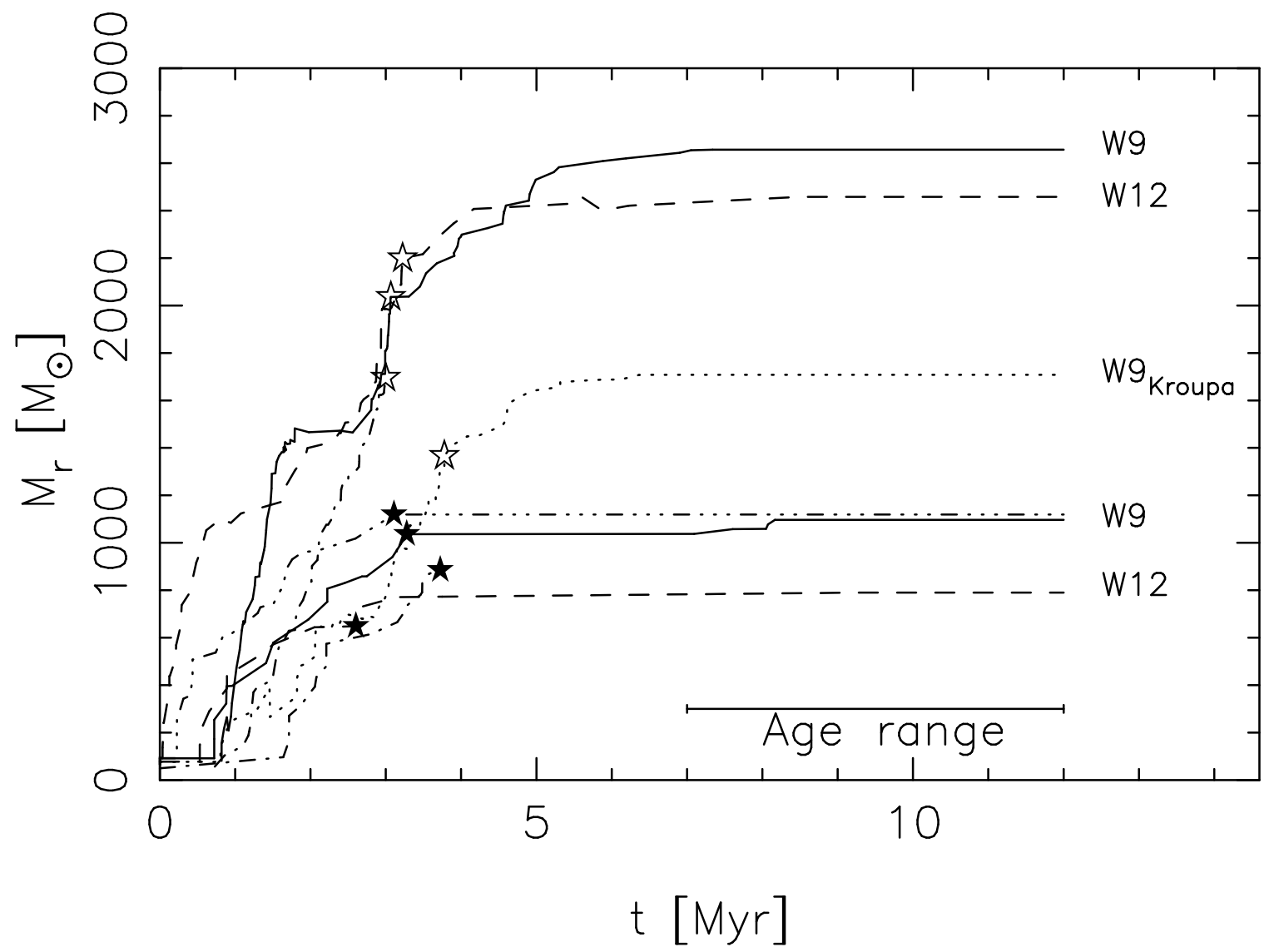

Figure 2: The growth in mass of the collision runaway star with time. The choice of initial concentration is labeled by the central potential, where W12(9) implies $W_{0}=12(9)$. For both choices, the top curves give the NBODY4 results, and the bottom curves the Starlab results. The runaway masses in NBODY4 are larger, since that code adopts larger stellar radii, as discussed in the Supplementary material. The star symbols indicate the moment when the runaway experiences a supernova, typically around $3 \mathrm{Myr}$. The open and filled stars indicate simulations performed with NBODY4 and Starlab, respectively. The solid and dashed curves show $M_{r}$ for a Salpeter ${ }^{2}$ IMF with a lower limit of $1 \mathrm{M}_{\odot}$ and $c \simeq 2.1\left(W_{0}=9\right)$ and $c \simeq 2.7\left(W_{0}=12\right)$. The dash-dotted curves are for two models with $W_{0}=9$ with an upper limit to the IMF of $50 \mathrm{M}_{\odot}$, instead of the standard $100 \mathrm{M}_{\odot}$ used in the other calculations; we terminated these runs at the moment the runaway star experiences a supernova. The dash-3-dotted curve shows the result for $W_{0}=12$ with a Salpeter IMF and with $10 \%$ primordial binaries. Finally, the dotted curve shows results for $W_{0}=9$ and a Kroupa $^{26} \mathrm{IMF}$ with a minimum mass of $0.1 \mathrm{M}_{\odot}$, in a simulation with 585,000 stars. The observed age range of MGG-11 and MGG-9 is indicated by the horizontal bar near the bottom of the figure. 


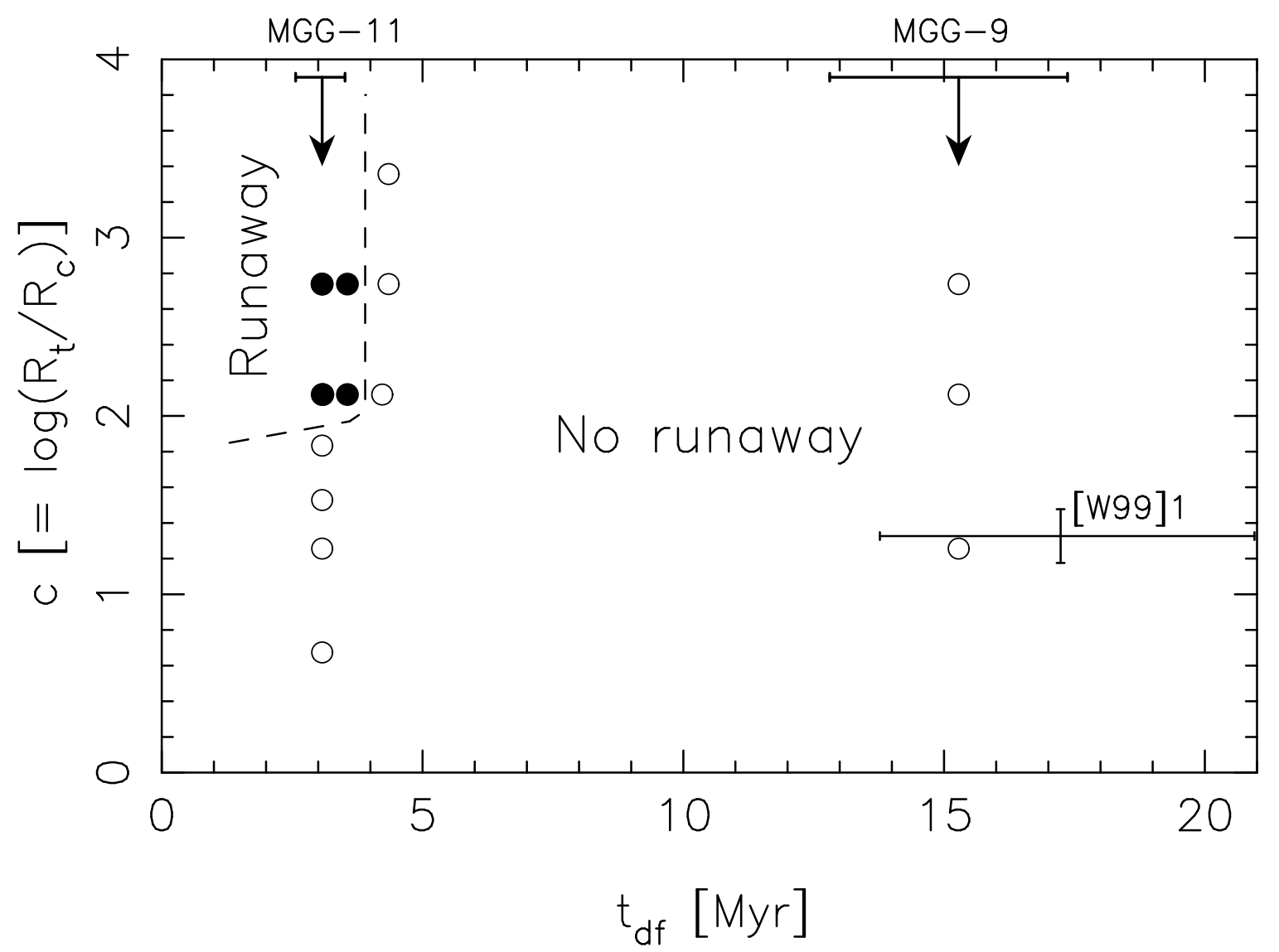

Figure 3: The area of parameter space for which runaway collision can occur, and where the process is prevented. Conditions for runaway merging identified through our simulations in the $\left\{t_{\mathrm{df}}, c\right\}$ plane, where $t_{\mathrm{df}}$ is the dynamical friction time scale for $100 \mathrm{M}_{\odot}$ stars, and $c$ the cluster concentration parameter. The horizontal error bars for the initial conditions for MGG-9 and MGG-11 reflect errors in the observed cluster mass and projected half light radius ${ }^{1}$. The best fit for the concentration parameter of the Antennae star cluster [W99] 1 is indicated by a vertical bar near $t_{\mathrm{df}} \simeq 17 \mathrm{Myr}$, the horizontal error bar reflect the uncertainty in the measured cluster mass and radius 9. Solid circles indicate simulations resulting in runaway merging, while open circles correspond to simulations in which no runaway merging occurred. The evolution of the mass of the runaway for the two leftmost filled circles are presented in Figure 2 , for a variety of initial conditions. Star clusters in the upper left corner enclosed by the dashed line are expected to host an IMBH formed by the runaway growth of a single star. In the other parts of the diagram (lower concentration and/or larger dynamical friction time) an IMBH cannot form by this process. 


\section{Supplementary material}

\section{This part of the paper will be published online and it has a separate bibliography}

In this section we discuss the selection of initial conditions, numerical methods, and the results of our simulations of the star clusters MGG-9 and MGG-11.

The first step in modeling this problem is to reconstruct the conditions under which MGG-11 was born $7-12 \mathrm{Myr}$ ago. McCrady et al ${ }^{1}$ determine the cluster age and mass-to-light ratio, and from these conclude that the mass function is consistent with a power-law having the Salpeter ${ }^{2}$ slope and a lower mass limit of $\sim 1 \mathrm{M}_{\odot}{ }^{1}$. At the current age of the cluster, all stars more massive than $\sim 17-25 \mathrm{M}_{\odot}{ }^{3}$ have experienced supernovae. We adopt this mass function in most of our simulations, with an upper limit of $100 \mathrm{M}_{\odot}$. This choice of mass limit is motivated by the fact that there are several clusters in the Milky Way Galaxy with characteristics similar to the two clusters studied here (MGG-11 and MGG-9) that are young enough for the highestmass stars still to be present. These clusters are the Arches ${ }^{4}$, Quintuplet ${ }^{5}$, NGC36036 and the central cluster R1367 in the 30 Doradus region in the large Magellanic cloud (LMC). The publications cited here support the existence of stars of $\gtrsim 100 \mathrm{M}_{\odot}$ in these clusters. In addition, an upper mass limit of $\sim 100 \mathrm{M}_{\odot}$ in the Galactic disc is probably quite realistic ${ }^{8}$.

The $1 \mathrm{M}_{\odot}$ lower mass cut-off for the initial mass function (IMF) is somewhat controversial. However, the mass functions in several of the young star clusters in the Antennae galaxies ${ }^{9}$, the Arches cluster ${ }^{4}$, Quintuplet cluster ${ }^{10}$, NGC1705-111 and the star cluster MGG-F in M8211 are consistent with a similar lower mass cut-off. For our study we adopt the observed mass function as a first choice, but in later runs we vary the mass function to test the effect of changing it.

At zero age the mean mass of our adopted IMF is $\langle m\rangle \simeq 3.1 M_{\odot}$. At the observed age of $7-12 \mathrm{Myr}$ the mean stellar mass has dropped to $\langle m\rangle \simeq 2.5-2.7 \mathrm{M}_{\odot}{ }^{3}$. What actually happens to stars of $\sim 17-25 \mathrm{M}_{\odot}$ which experience a supernova before $7-12 \mathrm{Myr}$ is not crucial for this estimate, as there are only 1500-2500 such stars. If we naively assume that all the mass in these stars is simply lost from the cluster we obtain mean stellar mass of $2.5-2.6 \mathrm{M}_{\odot}$. If we assume that stars more massive than $25 \mathrm{M}_{\odot}$ turn into $10 \mathrm{M}_{\odot}$ black holes and stars of lower mass into Chandrasekhar $\left[1.4 \mathrm{M}_{\odot}\right]$ mass neutron stars, the mean stellar mass drops to $\langle m\rangle \simeq 2.6-2.7 \mathrm{M}_{\odot}$. With today's measured mass of $M=3.5 \times 10^{5} \mathrm{M}_{\odot}{ }^{1}$ the cluster then contains $N=130,000-140,000$ stars, implying an initial total mass of $M_{0}=4.1-4.5 \times 10^{5} \mathrm{M}_{\odot}$.

In order to model the cluster we must know its initial half-mass radius $R$. We obtain this parameter by de-projecting the observed half-light radius and correcting for the expansion of the cluster during its lifetime. The two corrections are comparable, but with opposite sign: the half-mass radius is $\sim 25$ percent larger than the observed half-light radius, and mass loss due to stellar evolution drives adiabatic expansion of the cluster radius by $\sim 20$ percent. The initial half-mass radius of MGG-11 is then $R \simeq 1.2 \mathrm{pc}$. Here we assume that mass follows light after $12 \mathrm{Myr}$, i.e. that the light profile is not significantly affected by the segregation of the most massive stars. This turns out to be a reasonable assumption. Note that the mass-to-light ratio for 
MGG-11 reported by McCrady et al. is $L / M=3.5_{-1.5}^{+2.1}$, and that adding a $3000 \mathrm{M}_{\odot}$ non-luminous object to the cluster increases this value only by about 1 percent. A similar analysis for MGG-9 results in a total mass at birth of $M_{0} \simeq 1.8 \times 10^{6} \mathrm{M}_{\odot}$ and an initial half-mass radius of $R \simeq 2.6 \mathrm{pc}$.

We ignore the effect of the tidal field of M82 in our simulations. The runaway growth studied for these clusters takes place within the dynamical friction time scale of the most massive stars and the process discussed here takes place deep within the cluster core. Even though the cluster may lose a few stars to its host Galaxy, this does not affect the processes driving the collision runaway. Note that the two clusters MGG-9 and MGG-11 are close together on the sky, and could in principle be bound in a binary cluster. At the $3.9 \mathrm{Mpc}^{14}$ distance of $\mathrm{M} 82$, the projected distance between MGG-9 and MGG-11 is about 30 pc. We note that in the LMC there are a few hundred binary clusters for which the projected separation is smaller than $20 \mathrm{pc}$ 12,13 .

Our simulations were performed using two independently developed $N$-body packages, Starlab15, 16 and NBODY4 17, 18. Both codes achieve their highest speed when running on the GRAPE-6 special-purpose hardware 19, 20. A comparison between Starlab and NBODY4 is presented by Heggie (2001)21.

Both codes incorporate parametrized stellar evolution and allow for the possibility of direct physical collisions between stars, thus including the two key physical elements in our runaway scenario. The criteria for stellar collisions in both codes are quite similar; a collision occurs if the distance between two stars becomes smaller than the sum of the stellar radii. During a collision mass and momentum are conserved. These are reasonable assumptions since the relative velocity of any two colliding stars is typically much smaller than the escape speed from either stellar surface ${ }^{22}$. As the runaway star grows in mass its angular momentum increases due to off-axis collisions. Our stellar evolution prescriptions did not incorporate special treatments to account for this effect. Rapidly rotating stars tend to develop significant horizontal turbulence which may dramatically affect the further evolution of the star 23, 24. Calculations for a rapidly rotating star of $120 \mathrm{M}_{\odot}$ results in a $\sim 15$ percent extension of the lifetime compared with a non-rotating star 25 . This effect is stronger for lower mass stars, but has not yet been explored for more massive stars.

The collision radius of a star is taken to be identical to the stellar size, except for black holes for which we use the tidal radius, which is considerably larger than the Schwarzschild radius. The zero-age radii of various stellar evolution models are presented in Figure 5. The radii in Starlab are systematically smaller than those in NBODY4. The collision cross sections in the appropriate range of initial conditions are dominated by gravitational focusing, and therefore the stellar size propagates linearly in the collision rate, and consequently also in the final mass of the runaway star.

We simulated MGG-11 and MGG-9 using initial mass functions and half mass radii based on the observations of McCrady et al. ${ }^{1}$ Most of these calculations were performed with 131072 (128k) stars. The initial density distribution is poorly constrained by the observations and we therefore performed a series of simulations with various choices for the initial density profile, ranging from the very shallow $c \simeq 0.67$ (equivalent to $W_{0}=3$ ) to highly concentrated $c \simeq 3.4$ (equivalent to $W_{0}=15$ ). To 
further test our theoretical understanding that a collision runaway is prevented by the first supernova explosions for $t_{\mathrm{df}} \gtrsim 4 \mathrm{Myr}$ we systematically increased the initial $t_{\mathrm{df}}$ for two selected values of $c$ in our MGG-11 simulations (by varying the initial half-mass radius $R$ ), until the collision runaway was prevented. The results are presented in Figure 3 of the main text. Note that we plot the horizontal error bars for both clusters near the top of the figure for practical reasons; these should not be interpreted as upper limits on the concentration parameter.

In order to illustrate the early core collapse in the more concentrated models, and the absence thereof in the shallower models, Figure 4 plots the evolution of the core radius for a selected number of simulations for MGG-11. We discuss each curve in turn, starting at the top. The core radius of the shallow model, $c \simeq 0.67$ $\left(W_{0}=3\right)$, hardly changes with time. The intermediate model $c \simeq 1.8\left(W_{0}=8\right)$ almost experiences core collapse near $t=3 \mathrm{Myr}$ but as stellar mass loss starts to drive the expansion of the core it never really experiences collapse. Inspection of Figure 3 of the main text shows that neither of these clusters experienced a collision runaway. Core collapse occurs in the $c \simeq 2.1\left(W_{0}=9\right)$ model near $t=0.8 \mathrm{Myr}$, which also corresponds to the time of the first collision. The $c \simeq 2.7\left(W_{0}=12\right)$ simulation is so concentrated that it starts virtually in core collapse, and the entire cluster evolution is dominated by a post-collapse phase. Inspection of Figure 2 in the main text reveals that in these calculations the runaway also tends to start earlier. The larger fluctuations in the core radius for models $W_{0}=9$ and $W_{0}=12$ compared to the less concentrated models reflect the orbit of the runaway star around the density center of the star cluster. The runaway star is always found in the core, but occasionally at a substantial distance from the center.

We performed four simulations for MGG-11 adopting $W_{0}=9$ with different initial mass functions in order to study the effect of the mass function on the calculations. Reducing the upper limit on the IMF from $100 \mathrm{M}_{\odot}$ to $50 \mathrm{M}_{\odot}$ reduces the mass of the runaway star at the moment of the supernova explosion by about 20 percent. Simulations with the Kroupa (2001) ${ }^{26}$ IMF and a lower mass limit of $0.1 \mathrm{M}_{\odot}$, as well as one run with a Salpeter IMF and a lower mass limit of $0.2 \mathrm{M}_{\odot}$, resulted in a $\sim 30 \%$ lower runaway mass. These latter simulations were run with 585,000 stars. The lower mass of the runaway star in the simulations with the reduced upper mass limit is a direct consequence of the smaller number of massive stars in the simulation, causing the average mass of the collision counterparts to be smaller and the collision rate to be lower. We also performed a calculation using the initial conditions for MGG-11 but with the zero-age stellar radii published by Ishii et al. (1999) ${ }^{33}$ for the collision runaway. This calculation resulted in the a similar black hole mass as in the NBODY4 calculations with otherwise the same initial conditions.

In addition, we performed several simulations for MGG-9, which is well outside the regime where a collision runaway can occur (see Figure 3 of the main text). For MGG-9 the IMF is consistent with a Salpeter IMF with a lower mass limit of $0.2 \mathrm{M}_{\odot}$, and also with the Kroupa IMF. However, due to computational limitations we could only perform simulations with the $1 \mathrm{M}_{\odot}$ cut-off IMF for MGG-9. Simulations with the Kroupa IMF would require 3.6 million stars, which is beyond our current capabilities. In the case of the Salpeter IMF with a lower limit of $1 \mathrm{M}_{\odot}$, and with other 
parameters chosen consistent with the observations of McCrady et al for MGG-9, we did not observe runaway merging.

Finally, we performed two calculations for MGG-11 using $c \simeq 2.7\left(W_{0}=12\right)$, and including 10 percent primordial binaries. The current binary fractions for MGG-11 is not determined by the observations, but based on studies of other clusters, 10 percent seems a reasonable choice $27,28,29$. We select initial orbital separations ranging from Roche-lobe contact (at a binding energy of about $300 \mathrm{kT}$ ) to that corresponding to a binding energy of $\sim 3 \mathrm{kT}$, which is near the dividing line between hard and soft binaries ${ }^{30}$. (Note that the total kinetic energy of the stellar system is $\frac{3}{2} N k T$.) The other initial binary parameters were selected as in Portegies Zwart et al. (2001) 15. Though modest, this fraction should be sufficient to completely dominate the dynamical evolution of the cluster core ${ }^{31}$.

The presence of primordial binaries in our models causes an increase in the overall merger rate by about a factor of four. Most of these mergers do not involve the runaway star but occur as a result of unstable mass transfer in a primordial binary. The collision rate with the runaway star increases by about 30 percent, and as a consequence the mass of the runaway star at the time of supernova is 22-36 percent larger than without primordial binaries. We have neglected the possibility of primordial mass segregation, which would further favor the runaway process, as massive stars would be born preferentially in the cluster core.

1. McCrady, N., Gilbert, A. M., Graham, J. R. Kinematic masses of super star clusters in M82 from high-resolution near-infrared spectroscopy. Astrophys. J. 596, 240-252 (2003)

2. Salpeter, E. E. The luminosity function and stellar evolution. Astrophys. J. 121, 161167 (1955).

3. Eggleton, P. P., Fitchett, M. J., Tout, C. A. The distribution of visual binaries with two bright components. Astrophys. J. 347, 998-1011 (1989).

4. Figer, D. F. et al. Massive stars in the arches cluster. Astrophys. J. 581, 258-275 (2002).

5. Figer, D. F., McLean, I. S., Morris, M. Two new Wolf-Rayet stars and a luminous blue variable star in the Quintuplet (AFGL 2004) near the Galactic center. Astrophys. J. 447, L29-L33 (1995).

6. Nürnberger, D. E. A. Infrared observations of NGC 3603. III. The enigmatic, highly reddened sources of IRS 9. Astron. Astrophys. 404, 255-265 (2003).

7. Massey, P., Hunder, D. A. Star formation in R136: A cluster of $\mathrm{O} 3$ stars revealed by Hubble Space Telescope spectroscopy. Astrophys. J. 493, 180-194 (1998). 
8. Kroupa, P. \& Weitner, C. Galactic-field initial mass functions of massive stars. Astrophys. J. 598, 1076-1078 (2003)

9. Mengel, S, Lehnert, M. D., Thatte, N., Genzel, R. Dynamical masses of young star clusters in NGC 4038/4039. Astron. Astrophys. 383, 137-152 (2002)

10. Figer, D. F., McLean, I. S., Morris, M. Massive stars in the Quintuplet cluster. Astrophys. J. 514, 202-220 (1999)

11. Smith, L. J. \& Gallagher, J. S. M82-F: a doomed super star cluster? Mon. Not. R. Astron. Soc. 326, 1027-1040 (2001).

12. Bica, E. L. D., Schmitt, H. R., Dutra, C. M., Oliveira, H. L. A Revised and Extended Catalog of Magellanic System Clusters, Associations, and Emission Nebulae. II. The Large Magellanic Cloud. Astron. J. 117, 238-246 (1999).

13. Dieball, A., Müller, H., Grebel, E. K. A statistical study of binary and multiple clusters in the LMC. Astron. Astrophys. 391, 547-564 (2002).

14. Sakai, S. \& Madore, B. F. Detection of the red giant branch stars in M82 using the Hubble Space Telescope. Astrophys. J. 526, 599-606 (1999)

15. Portegies Zwart, S. F., McMillan, S. L. W., Hut, P., Makino, J. Star cluster ecology - IV. Dissection of an open star cluster: photometry. Mon. Not. R. Astron. Soc. 321, 199-226 (2001).

16. Hut, P., Makino, J., McMillan, S. L. W., Portegies Zwart S. Starlab. http://manybody.org/manybody/starlab.html (1995)

17. Aarseth, S. J. From NBODY1 to NBODY6: The growth of an industry. Pub. Astron. Soc. Pac. 111, 1333-1346 (1999).

18. Baumgardt, H., Makino, J. Dynamical evolution of star clusters in tidal fields. Mon. Not. R. Astron. Soc. 340, 227-246 (2003).

19. Makino, J., Taiji, M., Ebisuzaki, T., Sugimoto, D. GRAPE-4: A massively parallel special-purpose computer for collisional N-body simulations. Astrophys. J. 480, 432446 (1997).

20. Makino, J., Taiji, M., Ebisuzaki, T., Sugimoto, D. GRAPE-6: The massively parallel special-purpose computer for astrophysical particle simulations. submitted to Pub. Astron. Soc. Jap. http://arxiv.org/list/astro-ph/0310702 (2003).

21. Heggie, D. C. Astrophysical Supercomputing Using Particles IAU Symposium, Vol. 208, $2001 \mathrm{~J}$. Makino and P. Hut, eds. Two collaborative experiments in star cluster evolution. http://arxiv.org/list/astro-ph/0110021 (2001)

22. Lombardi, J. C. Jr., Warren, J. S., Rasio, F. A., Sills, A., Warren, A. R. Stellar collisions and the interior structure of blue stragglers. Astrophys. J. 568, 939-953 (2002). 
23. Sills, A., Faber, J. A., Lombardi, J. C. Jr., Rasio, F. A., Warren, A. R. Evolution of Stellar Collision Products in Globular Clusters. II. Off-Axis Collisions. Astrophys. J. 548, 323-334 (2001).

24. Maeder, A. Stellar rotation: Evidence for a large horizontal turbulence and its effects on evolution. Astron. Astroph. 399, 263-269 (2003).

25. Meynet, G. \& Maeder, A. Stellar evolution with rotation. X. Wolf-Rayet star populations at solar metallicity. Astron. Astroph. 404 975-990 (2003).

26. Kroupa, P. On the variation of the initial mass function. Mon. Not. R. Astron. Soc. 322, 231-246 (2001).

27. Albrow, M. D. et al. The frequency of binary stars in the core of 47 Tucanae. Astrophys. J. 559, 1060-1081 (2001).

28. Rubenstein, E. E. The search for main sequence binary stars in galactic globular clusters. Pub. Astron. Soc. Pac. 109, 933-933 (1997).

29. Yan, L. Binary stars in globular clusters. Thesis (PH.D.) CALIFORNIA INSTITUTE OF TECHNOLOGY, Dissertation Abstracts International, Volume: 57-02, Section: B, 0-1143 (1996)

30. Heggie, D. Binary evolution in stellar dynamics. Mon. Not. R. Astron. Soc. 173, 729-787 (1975).

31. McMillan, S. L. W., Hut, P., Makino, J., Star cluster evolution with primordial binaries. II - Detailed analysis. Astrophys. J. 372, 111-124 (1991)

32. Hurley, J. R., Pols, O. R., Tout, C. A. Comprehensive analytic formulae for stellar evolution as a function of mass and metallicity. Mon. Not. R. Astron. Soc. 315, 543-369 (2000).

33. Ishii, M., Ueno, M., Kato, M. Core-halo structure of a chemically homogeneous massive star and bending of the zero-age main sequence. Pub. Astron. Soc. Jap. 51, 417-424 (1999).

34. Stothers, B., Chin, C-W. On two theories of the cyclical outbursts of eta Carinae. Astrophys. J. 489, 319-330 (1997). 


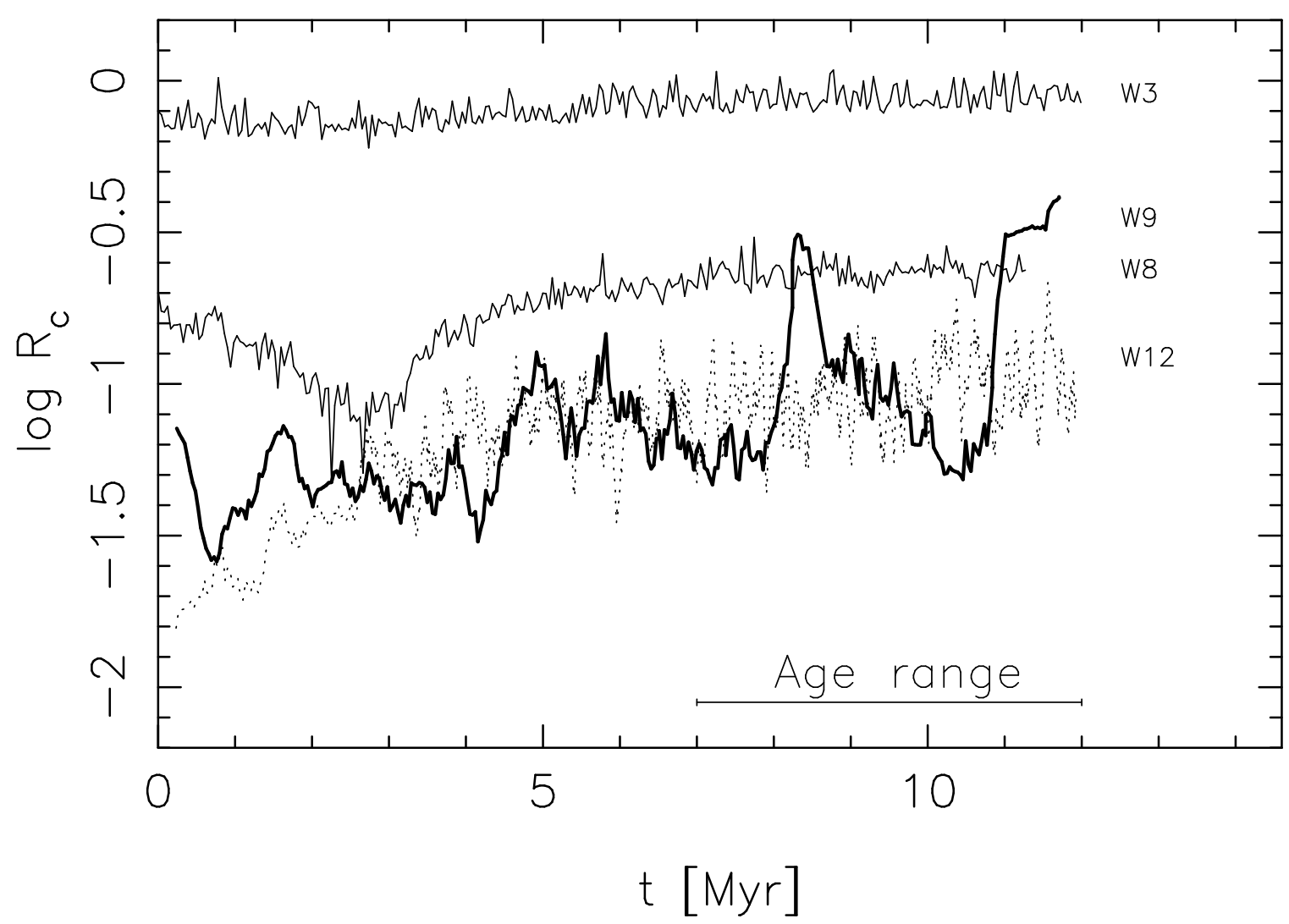

Figure 4: Evolution of the core radius for four simulations of the star cluster MGG11. These calculations are performed with Starlab with $c \simeq 0.67\left(W_{0}=3\right), c \simeq 1.8$ $\left(W_{0}=8\right), c \simeq 2.1\left(W_{0}=9\right.$, bold curve $)$ and $c \simeq 2.7\left(W_{0}=12\right.$, dotted curve $)$ indicated along the right edge of the figure as $W 3, W 8, W 9$, and $W 12$ respectively. The W9 curve is plotted with a heavy line to distinguish it from the curves for W8 and W12. The age range of the observed clusters is indicated near the bottom of the figure. The large variation in the core radius for the $W_{0}=9$ and $W_{0}=12$ models is caused in part by the presence of the runaway star. 


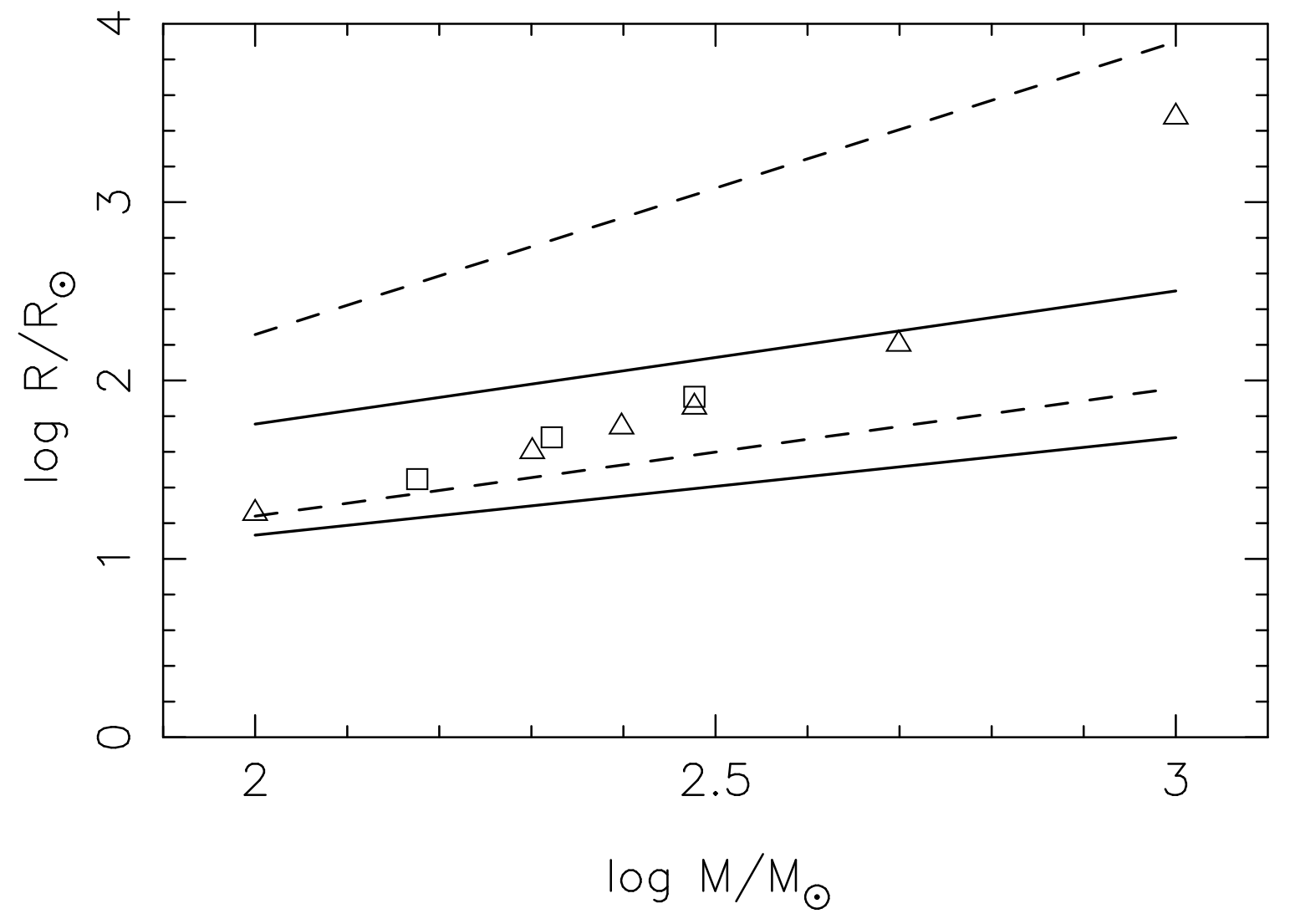

Figure 5: Stellar radius as a function of zero-age mass for various models of highmass stars. The lower and upper solid lines indicate the radii of zero-age and terminal age main-sequence stars from the stellar evolution model used in Starlab, which are based on the prescription of Eggleton et al. (1989) ${ }^{3}$. The dashed lines are taken from the stellar evolution prescription used in NBODY4 which are based on the evolutionary calculation by Hurley et al. (2000) ${ }^{32}$. The triangles are from the calculations of Ishii et al. (1999) ${ }^{33}$, the squares are taken from Stothers (1997) ${ }^{34}$. 